\title{
Negative spectra of elliptic operators
}

\author{
Stanislav Molchanov • Oleg Safronov
}

Received: 8 April 2012 / Accepted: 20 April 2012 / Published online: 9 May 2012

(C) The Author(s) 2012. This article is published with open access at SpringerLink.com

\begin{abstract}
We establish different estimates for the sums of negative eigenvalues of elliptic operators. Our proofs are based on a property of the eigenvalue sums that might be viewed as a certain convexity with respect to the perturbation.
\end{abstract}

\section{Estimates for the discrete spectrum}

We are going to establish an estimate for sums of negative eigenvalues of elliptic operators. Our proof is based on the convexity of the eigenvalue sums. It turns out that such a property can be formulated for arbitrary self-adjoint operators. Let $A$ be a positive self-adjoint operator and let $-V$ be a self-adjoint perturbation of $A$. Consider the operator

$$
H=A-V, \quad V \geq 0 .
$$

In most of our applications, the operator

$$
V^{1 / 2}(A+I)^{-1 / 2} \in \mathfrak{S}_{\infty}
$$

will be compact. This condition implies that the negative spectrum of $H$ is discrete. In that case, we study the negative eigenvalues $\lambda_{j}$ of the operator $H$. Sometimes we will indicate their dependence on $V$ using the notation:

Communicated by A. Laptev.

S. Molchanov · O. Safronov $(\varangle)$

Mathematics and Statistics, UNC Charlotte, 9201 University City Blvd,

Charlotte, NC 28223, USA

e-mail: osafrono@uncc.edu 


$$
\lambda_{j}=\lambda_{j}(V), \quad j=1,2, \ldots
$$

However, condition (1.1) is violated and the operator

$$
V^{1 / 2}(A+I)^{-1 / 2} \in \Re
$$

is only bounded, then we study the bottom of the spectrum

$$
\lambda_{1}=\inf \sigma(H)
$$

of the operator $H$ which might be either an eigenvalue or a point of accumulation of the set $\sigma(H)$. Let now $V_{1}$ and $V_{2}$ be two positive selfadjoint operators, such that

$$
V=V_{1}+V_{2}
$$

Lemma 1.1 (Convexity) Let $V, V_{1}$ and $V_{2}$ satisfy (1.2). Let $\lambda_{j}(V)$ and $\lambda_{j}\left(V_{n}\right)$ be enumerated in the increasing order:

$$
\lambda_{1} \leq \lambda_{2} \leq \cdots
$$

Then for any $p \geq 1$, and $\varepsilon \in(0,1)$, the eigenvalue sum

$$
S(V)=\sum_{j=1}^{n}\left|\lambda_{j}\right|^{p}
$$

has the property that

$$
S(V) \leq \varepsilon S\left(\varepsilon^{-1} V_{1}\right)+(1-\varepsilon) S\left((1-\varepsilon)^{-1} V_{2}\right), \quad 0<\varepsilon<1 .
$$

If (1.1) does not hold, then

$$
\lambda_{1}(V) \geq \varepsilon \lambda_{1}\left(\varepsilon^{-1} V_{1}\right)+(1-\varepsilon) \lambda_{1}\left((1-\varepsilon)^{-1} V_{2}\right), \quad 0<\varepsilon<1 .
$$

with $\lambda_{1}(V)$ and $\lambda_{1}\left(V_{n}\right)$ defined by $\lambda_{1}(V)=\inf \sigma(H)$ and $\lambda_{1}\left(V_{n}\right)=\inf \sigma\left(A-V_{n}\right)$. Proof Consider the case $p=1$. In this case,

$$
S_{n}(V):=\sum_{1}^{n}\left|\lambda_{j}\right|=-\min _{P_{n}} \operatorname{tr} H P_{n}
$$

where the minimum is taken over all possible orthogonal projections $P_{n}$ of rank $n$ that have the property $\operatorname{Ran} P_{n} \subset D(H)$.

Since

$$
H=\varepsilon\left(A-\varepsilon^{-1} V_{1}\right)+(1-\varepsilon)\left(A-(1-\varepsilon)^{-1} V_{1}\right),
$$


we obtain that

$$
\operatorname{tr} H P_{n}=\varepsilon \operatorname{tr}\left(A-\varepsilon^{-1} V_{1}\right) P_{n}+(1-\varepsilon) \operatorname{tr}\left(A-(1-\varepsilon)^{-1} V_{1}\right) P_{n}
$$

This implies (1.3) for $p=1$.

A standard convexity argument allows one to pass from the case $p=1$ to the case $p>1$. We have established already that

$$
\begin{aligned}
& \sum_{1}^{n}\left(\left|\lambda_{j}(V)\right|-\gamma\right)_{+} \leq \sum_{1}^{n} \varepsilon\left(\left|\lambda_{j}\left(\varepsilon^{-1} V\right)\right|-\gamma\right)_{+} \\
& \quad+\sum_{1}^{n}(1-\varepsilon)\left(\left|\lambda_{j}\left((1-\varepsilon)^{-1} V\right)\right|-\gamma\right)_{+},
\end{aligned}
$$

where $(\cdot)_{+}$denotes the operation of taking the positive part of a number. It remains to observe that

$$
\sum_{1}^{n}\left|\lambda_{j}\right|^{p}=c \int_{0}^{\infty} \sum_{1}^{n}\left(\left|\lambda_{j}(V)\right|-\gamma\right)_{+} \gamma^{p-2} d \gamma
$$

where

$$
c^{-1}=\int_{0}^{\infty} \sum_{1}^{n}(1-\gamma)_{+} \gamma^{p-2} d \gamma
$$

The proof is completed.

Applications. Consider some applications of Lemma 1.1. Let $A$ be the selfadjoint operator in $L^{2}\left(\mathbb{R}^{d}\right)$ defined by

$$
A=(-\Delta)^{l}, \quad 2 l>d,
$$

and let $V$ be the operator of multiplication by a positive function $V(x) \geq 0$.

Theorem 1.1 Let $2 l>d$ and let $p \geq 1$. Then the negative eigenvalues $\lambda_{j}$ of the operator $(-\Delta)^{l}-V$ satisfy the inequality

$$
\left(\sum_{j}\left|\lambda_{j}\right|^{p}\right)^{\frac{2 l-d}{2 l p}} \leq(2 \pi)^{-d} \int_{\mathbb{R}^{d}} V(x) d x \int_{\mathbb{R}^{d}} \frac{d \xi}{|\xi|^{2 l}+1} .
$$

In particular, the negative eigenvalues $\lambda_{j}$ of the operator

$$
-\frac{d^{2}}{d x^{2}}-V, \quad V>0
$$


satisfy the estimate

$$
\left(\sum_{j}\left|\lambda_{j}\right|^{p}\right)^{\frac{1}{2 p}} \leq \frac{1}{2} \int_{\mathbb{R}} V(x) d x, \quad p \geq 1 .
$$

Proof Let us prove this statement for $p=1$. The case $p>1$ is similar. Assume for the moment that $V=\sum_{j} h_{j} \phi\left(x-x_{j}\right)$, where the positive function $\phi$ has the property

$$
\int \phi(x) d x=1
$$

Using the convexity of the eigenvalue sum we obtain:

$$
S(V) \leq \sum_{j} \varepsilon_{j} S\left(\varepsilon_{j}^{-1} h_{j} \phi\right), \quad \sum \varepsilon_{j}=1 .
$$

Now, choosing

$$
\varepsilon_{j}=\frac{h_{j}}{\sum_{j} h_{j}}
$$

we derive the inequality

$$
S(V) \leq S(\alpha \phi), \quad \text { where } \alpha=\int_{\mathbb{R}^{d}} V(x) d x
$$

Repeating this operation over and over, we will obtain a similar inequality with $\phi(x)=$ $\delta(x)$. In this case $S(\alpha \phi)$ can be easily computed, because the operator $(-\Delta)^{l}-\alpha \delta(x)$ has exactly one eigenvalue below zero.

Remark 1 If $d=l=1$, this statement follows from the celebrated result of Hundertmark et al. [3] saying that

$$
\sum_{j}\left|\lambda_{j}\right|^{1 / 2} \leq \frac{1}{2} \int_{\mathbb{R}} V(x) d x, \quad d=1 .
$$

Remark 2 The constant in (1.5) is sharp, because if $V=\delta(x)$, then the operator $(-\Delta)^{l}-V$ has exactly one eigenvalue $\lambda_{1}$ such that

$$
\left|\lambda_{1}\right|^{\frac{2 l-d}{2 l}}=(2 \pi)^{-d} \int_{\mathbb{R}^{d}} \frac{d \xi}{|\xi|^{2 l}+1} .
$$


Consider the matrix-valued case, when $A$ is the selfadjoint operator in $L^{2}\left(\mathbb{R}^{d} ; \mathbb{C}^{n}\right)$ defined by

$$
A=(-\Delta)^{l} I, \quad 2 l>d
$$

and $V$ is an $n \times n$-matrix valued potential. We shall assume that $(V(x) \eta, \eta) \geq 0$ for each $x \in \mathbb{R}^{d}$ and each vector $\eta \in \mathbb{C}^{n}$. The importance of such problems with a matrix-valued potentials was discovered by Laptev and Weidl [4]. Using the technique of [4], one can reduce the study of the Schrödinger operator in the multi-dimensional case to the case $d=1$. As a result, one obtains the best constants in the Lieb-Thirring inequalities.

Theorem 1.2 Let $2 l>d$ and let $p \geq 1$. Then the negative eigenvalues $\lambda_{j}$ of the operator $(-\Delta)^{l} I-V$ satisfy the inequality

$$
\left(\sum_{j}\left|\lambda_{j}\right|^{p}\right)^{\frac{2 l-d}{2 l p}} \leq(2 \pi)^{-d} \int_{\mathbb{R}^{d}} \operatorname{tr} V(x) d x \int_{\mathbb{R}^{d}} \frac{d \xi}{|\xi|^{2 l}+1} .
$$

Proof We apply the same method as before, but take into account the fact that $V(x)$ is a matrix. Again, we prove this statement only for $p=1$, because the case $p>1$ is similar. Assume for the moment that $V=\sum_{j} h_{j} \phi\left(x-x_{j}\right) P_{j}$, where the positive function $\phi$ has the property (1.6) and $P_{j}$ are orthogonal projections in $\mathbb{C}^{n}$ of rank 1. Using the convexity of the eigenvalue sum we obtain:

$$
S(V) \leq \sum_{j} \varepsilon_{j} S\left(\varepsilon_{j}^{-1} h_{j} \phi P_{j}\right), \quad \sum \varepsilon_{j}=1 .
$$

Now, selecting

$$
\varepsilon_{j}=\frac{h_{j}}{\sum_{j} h_{j}}
$$

we derive the inequality

$$
S(V) \leq S(\alpha \phi P), \quad \text { where } \quad \alpha=\int_{\mathbb{R}^{d}} \operatorname{tr} V(x) d x
$$

and $P$ is an orthogonal projection of rank 1 . Repeating this operation over and over, we will obtain a similar inequality with $\phi(x)=\delta(x) P$. In this case $S(\alpha \phi P)$ can be easily computed, because the operator $(-\Delta)^{l} I-\alpha \delta(x) P$ has exactly one eigenvalue below zero.

Theorem 1.3 Let $\lambda_{j}$ be the negative eigenvalues of the operator

$$
-\frac{d^{2}}{d x^{2}}-V, \quad V>0
$$


with Dirichlet boundary conditions on the boundary of the interval $[0, L]$. Suppose that

$$
\int_{-\infty}^{\infty} V(x) d x>4 / L
$$

Then

$$
\sum_{j}\left|\lambda_{j}\right| \leq \beta\left[\int_{0}^{L} V(x) d x\right]
$$

where $\beta[t]$ is the function satisfying the equation

$$
2 \frac{\sqrt{\beta} \cosh (L \sqrt{\beta} / 2)}{\sinh (L \sqrt{\beta} / 2)}=t, \quad t>4 / L
$$

The proof is very simple. One has to minimize the lowest eigenvalue of the operator

$$
-\frac{d^{2}}{d x^{2}}-\alpha \delta\left(x-x_{0}\right), \quad x_{0} \in[0, L], \quad \alpha=\int_{0}^{L} V d x
$$

by varying $x_{0}$. By the Birman-Schwinger principle the lowest eigenvalue $\lambda$ of this operator operator is the solution of the equation

$$
\alpha G_{\lambda}\left(x_{0}, x_{0}\right)=1
$$

where $G_{\lambda}(x, y)$ is the integral kernel of the operator $\left(-d^{2} / d x^{2}-\lambda\right)^{-1}$. The minimum of $\lambda$ is attained at the middle point $x_{0}=L / 2$. It remains to note that (1.8) with $\lambda=-\beta$ and $x_{0}=L / 2$ is equivalent to (1.7) with $t=\alpha$.

Remark If

$$
\int_{0}^{L} V(x) d x \leq 4 / L, \quad V>0
$$

then the operator

$$
-\frac{d^{2}}{d x^{2}}-V
$$

with Dirichlet boundary conditions on the boundary of the interval $[0, L]$ does not have negative eigenvalues. 
Theorem 1.4 Let $\lambda_{j}$ be the negative eigenvalues of the operator

$$
-\frac{d^{2}}{d x^{2}}-V, \quad V>0
$$

with Neumann boundary conditions on the boundary of the interval $[0, L]$. Then

$$
\sum_{j}\left|\lambda_{j}\right| \leq \gamma\left[\int_{0}^{L} V(x) d x\right]
$$

where $\gamma[t]$ is the function satisfying the equation

$$
\frac{\sqrt{\gamma} \sinh (L \sqrt{\gamma})}{\cosh (L \sqrt{\gamma})}=t, \quad t>0
$$

Proof Again, one has to minimize the lowest eigenvalue of the operator

$$
-\frac{d^{2}}{d x^{2}}-\alpha \delta\left(x-x_{0}\right), \quad x_{0} \in[0, L], \quad \alpha=\int_{0}^{L} V d x
$$

by varying $x_{0}$. However, the optimal choice of $x_{0}$ will be now different because of the Neumann boundary conditions. By the Birman-Schwinger principle the lowest eigenvalue $\lambda$ of this operator operator is the solution of the equation

$$
\alpha G_{\lambda}\left(x_{0}, x_{0}\right)=1
$$

where $G_{\lambda}(x, y)$ is the integral kernel of the operator $\left(-d^{2} / d x^{2}-\lambda\right)^{-1}$. The minimum of $\lambda$ is attained at the edges of the interval $[0, L]$. It remains to note that (1.10) with $\lambda=-\gamma$ and $x_{0}=0$ is equivalent to (1.9) with $t=\alpha$.

\section{Birman's theorem}

As we mentioned before, the bottom of the spectrum of the operator $H$ does not have to be an eigenvalue. It might be a point of the essential spectrum of $H$. Still, $\lambda_{1}=\inf \sigma(H)$ has the same property as $S(V)$ : it is convex in the following sense:

$$
\left|\lambda_{1}\left(V_{1}+V_{2}\right)\right| \leq \varepsilon\left|\lambda_{1}\left(\varepsilon^{-1} V_{1}\right)\right|+(1-\varepsilon)\left|\lambda_{1}\left((1-\varepsilon)^{-1} V_{2}\right)\right|
$$

Combining this property with the possibility to impose Neumann conditions, we obtain the following statement.

Theorem 2.1 Let $H$ be the one-dimensional Schrödinger operator

$$
H=-\frac{d^{2}}{d x^{2}}-V(x), \quad V \geq 0,
$$


with a potential satisfying

$$
\int_{n}^{n+1} V(x) d x \leq \alpha, \quad \forall n \in \mathbb{Z} .
$$

Then the bottom of the spectrum of $H$ satisfies the estimate

$$
\inf \sigma(H) \geq-\gamma[\alpha]
$$

where $\gamma[t]$ is defined by

$$
\frac{\sqrt{\gamma} \sinh (\sqrt{\gamma})}{\cosh (\sqrt{\gamma})}=t, \quad t>0 .
$$

Proof By imposing the Neumann boundary conditions at integer points $n \in \mathbb{Z}$, we reduce this theorem to the estimate of the lowest eigenvalue of the operator on the interval $[n, n+1]$ with Neumann boundary conditions at the edges. But this has been already done in Theorem 1.4.

Theorem 2.1 could be viewed as a version of the following result proven by Birman. Such a statement was mentioned in [2], but we believe that the full credit can be already given to the earlier paper [1].

Theorem 2.2 Let $H$ be the one-dimensional Schrödinger operator

$$
H=-\frac{d^{2}}{d x^{2}}-V(x), \quad V \geq 0
$$

whose domain of definition contains all $C_{0}^{\infty}$-functions. Then $H$ is semi-bounded from below if and only if

$$
\sup _{n} \int_{n}^{n+1} V(x) d x<\infty \text {. }
$$

The proof of Theorem 2.1 seems to be simple and natural. Besides that, this theorem is sharp, which means that the function $\gamma[\alpha]$ can not be made smaller.

Theorem 2.3 Let $\alpha>0$ be a given positive number. For any $\varepsilon>0$, there exists a positive function $V$ with

$$
\int_{n}^{n+1} V(x) d x=\alpha, \quad \forall n \in \mathbb{Z},
$$


such that bottom of the spectrum of the one-dimensional Schrödinger operator

$$
H=-\frac{d^{2}}{d x^{2}}-V(x)
$$

satisfies the estimate

$$
-\gamma[\alpha] \leq \inf \sigma(H) \leq-\gamma[\alpha]+\varepsilon
$$

where $\gamma[t]$ is defined by (2.1).

Proof Indeed, set

$$
V=2 \alpha \sum_{n \in \mathbb{Z}} \phi_{\epsilon}(x-2 n), \quad 0<\epsilon<1 / 2,
$$

where

$$
\phi_{\epsilon}(x)= \begin{cases}0, & \text { if }|x-1|>\epsilon \\ \frac{1}{2 \epsilon}, & \text { if }|x-1| \leq \epsilon\end{cases}
$$

This potential is an even periodic function, therefore the ground state $\psi$, defined as the unique periodic solution of the equation

$$
-\psi^{\prime \prime}(x)-V \psi(x)=\lambda_{1} \psi(x), \quad \lambda_{1}=\inf \sigma(H)
$$

is also even. Consequently, it satisfies the Neumann boundary condition at $x=0$. Moreover, $\psi(x-1)$ is even as well. It implies that $\psi^{\prime}(n)=0$ at all points $n \in \mathbb{Z}$. Therefore we conclude that $\lambda_{1}$ coincides with the lowest eigenvalue of the operator $-d^{2} / d x^{2}-V(x)$ with Neumann boundary conditions at the edges of the interval $[0,1]$. This eigenvalue was studied in the proof of Theorem 1.4, according to which $\lambda_{1} \rightarrow \gamma[\alpha]$ as $\epsilon \rightarrow 0$.

Open Access This article is distributed under the terms of the Creative Commons Attribution License which permits any use, distribution, and reproduction in any medium, provided the original author(s) and the source are credited.

\section{References}

1. Birman, M.S.: Perturbations of quadratic forms and the spectrum of singular boundary value problems. Dokl. Akad. Nauk SSSR 125, 471-474 (1959, in Russian)

2. Birman, M.Sh.: Discrete spectrum in the gaps of a continuous one for perturbations with large coupling constants. Adv. Soviet Math. 7, 57-73 (1991)

3. Hundertmark, D., Lieb, E., Thomas, L.: A sharp bound for an eigenvalue moment of the one-dimensional Schrödinger operator. Adv. Theor. Math. Phys. 2, 719-731 (1998)

4. Laptev, A., Weidl, T.: Sharp Lieb-Thirring inequalities in high dimensions. Acta Math. 184(1), 87-111 (2000) 